\title{
Editorial
}

\section{Patients at a high risk of infectious endocarditis: What's up Doc?}

\author{
Philippe Lesclous*
}

\begin{abstract}
"Do what you can, with what you have, where you are"
Theodore Roosevelt (26th President of the United States of America, 1856-1919)

Oral management of patients at risk of infectious endocarditis (IE) has evolved considerably over the past 10 years to a point where it raises many questions or even misconceptions among some professionals in the oral care domain.
\end{abstract}

In 2007, based on the risk/benefit ratio (or cost/ effectiveness), the American Heart Association (AHA) limited the use of a single-dose antibiotic prophylaxis to the hour before hemorrhagic intervention, particularly oral, only to patients with high IE risk [1].

In 2009, the European Society of Cardiology (ESC) endorsed the recommendations of the AHA, further specifying the antibiotic prophylaxis protocol [2]. In 2011, the National Agency for Safety of Medicines (ANSM) endorsed the recommendations of the ESC by specifying the authorized and contraindicated oral procedures for patients at high IE risk [3]. In 2015, ESC revised its 2009 recommendations and opened the door for dental implant procedures for patients at high IE risk [4]. In 2017, a multidisciplinary task force (cardiologists, infectious disease specialists, oral surgeons, periodontists, and endodontists) was set up under the auspices of the appropriate scientific societies, with the mission of defining the oral management of patients at high IE risk, especially prosthetic valve carriers (who represent the vast majority of these patients), focusing first on implant practice. This work group endorsed the 2015 ESC recommendations, but stated that

- Placement of dental implants remains contraindicated in patients with history of IE,

- A delay of at least 3 months after the prosthetic valve surgery should be observed,
- This surgical practice is elaborated and should to comply with strict operating asepsis conditions and had to take into account certain general and local factors, including the choice of implant [5].

Beyond these, this task force should also clarify the feasibility of other hemorrhagic and therefore bacteremic oral procedures that are always contraindicated in this population (endodontic treatment or re-treatment, periapical surgery, periodontal surgery, or pre- and peri-implant surgeries), in the light of current scientific literature.

These successive changes over a short period intrigue or even offend some oral healthcare professionals, and this is understandable as the changes are radical but always for the benefit of the involved patients.

Today, it is time to end all quarrels, to give up opinions that are more or less clear and circumstantial, and more generally to avoid the realm of belief, all with such damaging effects. It is time to broaden and update the composition of this work group to include all concerned healthcare professionals and give it the opportunity to work under the auspices of a national health agency (ANSM or the French National Authority for Health; HAS), with all that this implies on the methodological level and the seriousness in the approach. This will probably not be enough to rally the most skeptical and derisive, but it should still reassure many of those and should also improve the oral care provided to these high IE risk patients.

As such, the French Society of Oral Surgery-our societyhas a pivotal role to play.

\section{References}

1. Wilson W, Taubert KA, Gewitz M et al. Prevention of infective endocarditis: Guidelines from the American Heart Association. Circulation 2007;116:1736-1754.

2. Habib G, Hoen B, Tornos P et al. Guidelines on the prevention, diagnosis, and treatment of infective endocarditis (new version 2009): the Task Force on the Prevention, Diagnosis, and Treatment of Infective Endocarditis of the European Society of Cardiology (ESC). Eur Heart J 2009;30:2369-2413.

\footnotetext{
*Correspondence: philippe.lesclous@univ-nantes.fr
} 
3. Agence Nationale de Sécurité du Médicament et des produits de santé. Prescription des antibiotiques en pratique buccodentaire. ANSM 2011; http://ansm.sante.fr/Mediatheque/Publica tions/Recommandations-Medicaments.

4. Habib G, Lancellotti P, Antunes MJ et al. 2015 ESC Guidelines for the management of infective endocarditis: The Task
Force for the Management of Infective Endocarditis of the European Society of Cardiology (ESC). Eur Heart J 2015;36: 3075-3123.

5. Millot S, Lesclous $\mathrm{P}$, Colombier ML et al. Position paper for the évaluation and management of oral status in patients with valvular disease. Arch Cardiovasc Dis 2017;110:482-494. 